\title{
CARCINOMA DE CUEILO UTERINO EN EL HOSPITAL UNIVERSITARIO SAN VICENTE DE PAUL - MEDELLIN
}

\author{
Dr. Jaime Botero Uribe* \\ Dr. Franco Barros Pabón**
}

\section{INTRODUCCION}

Según el Registro Nacional de Cáncer (1), en Colombia ocurrieron en 19642.005 casos de carcinoma del aparato genital femenino, lo cual representa el $26,7 \%$ de los casos de cáncer diagnosticados en Colombia en ese año. De estos, 1.664 o sea el $83 \%$ estaban localizados en el cuello uterino, y la gran mayoría, un $83,8 \%$ eran carcinomas epidermoides.

Según las estadísticas del D.A.N.E. (2) en 1967 hubo en Colombia 1.764 casos de carcinoma del cuello uterino y 459 casos de muerte por la misma entidad.

Desde principios del presente siglo se ha considerado el cáncer del cuello uterino como el de más frecuente ocurrencia en la población de Antioquia (3). En este Departamento ocurrieron en 1967, 92 defunciones debido a la entidad mencionada (4).

Correa (5) analizó los resultados histológicos de las biopsias estudiadas en el Instituto de Anatomía Patológica de la Facultad de Medicina de la Universidad de Antioquia entre los años de 1944 y 1951, y encontró que el cáncer del cuello uterino ocupaba el primer lugar entre las pacientes del sexo femenino y el $2^{\circ}$, dspués del de piel, en el grupo conjunto de hombres y mujeres.

Considerando solamente la ciudad de Medellín, el cáncer en general ocupaba el 4: lugar entre las causas de muerte en 1943, y el del útero estaba entre las cuatro más frecuentes en cuanto a localización (6).

A pesar de que desde hace más de veinte años se viene practicando en el Hospital Universitario San Vicente de Paúl el tratamiento para el carcinoma de cuello uterino mediante cirugía o radioterapia, independientes o combinadas, hasta el presente no se ha intentado una evaluación de los resultados de esa terapia que permita conocer el grado de su eficacia, ni se ha determinado la supervivencia a los cinco años de las pacientes que se han podido controlar.

\section{Objetivos}

Nos proponemos en el presente trabajo:

* Del Departamento de Obstetricia y Ginecología. Facultad de Medicina. Universidad de Antioquia.

** Interno Rotatorio, Hospital Universitario San Vicente de Paúl. Actualmente Residente del Departamento de Ginecología y Obstetricia del Hospital Universitario, San Ignacio. Bogotá. 
1) Determinar la edad, paridad y grado clínico del carcinoma de cuello, en una muestra de las pacientes que consultaron por primera vez al Hospital entre 1954-1959.

2 ) Correlacionar la edad con el grado clínico de la enfermedad.

3 ) Determinar los métodos de tratamiento que se han empleado en la época que comprende el estudio.

4) Averiguar los resultados de dicho tratamiento, en cuanto a tasa de curaciones a los cinco años, mediante determinación del número de pacientes que pudieron seguirse por ese lapso.

\section{Material y Métodos}

Se determinó en primer lugar el número de pacientes con diagnóstico de carcinoma de cuello uterino que figuraban en la Sección de Estadística del Hospital Universitario San Vicente de Paúl y se encontró que en el período de estudio había 607 pacientes. Se tomó entonces, por el sistema aleatorio, una muestra representativa de este universo, seleccionando la historia de una de cada tres pacientes de las que figuraban en la lista. Se analizó cada historia en relación a los objetivos mencionados y se pasaron los hallazgos a tarjetas, para tabulación. Inicialmente se fijó la muestra en 196 historias, pero al determinar la significación estadística que tendría, se observó que para ello necesitaban agregarse 40 más, lo cual se hizo escogiéndolas igualmente por un método de muestreo al azar.

\section{Resultados}

El Cuadro 1 muestra el grado clínico que presentaban las 236 pacientes cuyas historias se analizaron. Se observa que la gran mayoría pertenecía a los grados II y III, lo cual se explica fácilmente por el hecho de que acuden a la consulta solamente en la fase claramente sintomática de la enfermedad.

\section{CUADRO 1}
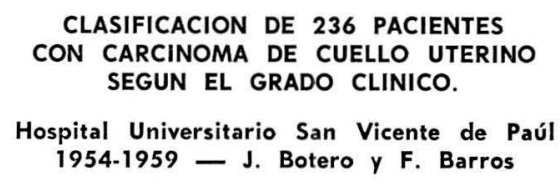

\begin{tabular}{|c|c|c|c|}
\hline Grado Clínico & $\mathbf{N}^{0} \mathrm{~d}$ & de Pacientes & $\%$ \\
\hline 0 & & 12 & 5 \\
\hline 1 & & 19 & 8 \\
\hline II & & 64 & 27,1 \\
\hline III & & 134 & 56,7 \\
\hline IV & & 7 & 2,9 \\
\hline TOTAL & & 236 & 100,0 \\
\hline
\end{tabular}

El Cuadro 2 indica que los grupos de edades entre los 31 y los 60 años comprendían la gran mayoría de las pacientes. En el Cuadro 3 se observa que la gran multiparidad era igualmente el factor acompañante en una alta proporción de las mismas.

\section{CUADRO 2}

\section{CLASIFICACION DE 236 PACIENTES \\ CON CARCINOMA DE CUELLO UTERINO SEGUN GRUPOS DE EDADES.}

\begin{tabular}{|c|c|c|c|c|}
\hline Grupo & de Edades & $\mathbf{N}^{\mathbf{9}}$ & de Pacientes & $\%$ \\
\hline 21 & $-\quad 30$ & & 14 & 5,9 \\
\hline 31 & $-\quad 40$ & & 59 & 25 \\
\hline 41 & 50 & & 81 & 34,3 \\
\hline $51-$ & $-\quad 60$ & & 59 & 25 \\
\hline $61-$ & $-\quad 70$ & & 19 & 8 \\
\hline $70 y$ & más & & 3 & 1,1 \\
\hline Descor & ocida & & 1 & 0,4 \\
\hline TOTAL & & & 236 & 100,0 \\
\hline
\end{tabular}




\section{CUADRO 3}

CLASIFICACION DE 236 PACIENTES

CON CARCINOMA DE CUELLO UTERINO SEGUN NUMERO DE PARTOS.

Hospital Universitario San Vicente de Paúl 1954-1959 - J. Botero y F. Barros

\begin{tabular}{lcr}
\hline Número de Partos & No de Pacientes & $\%$ \\
\hline 0 & 6 & 2,5 \\
$1-2$ & 21 & 8,8 \\
$3-4$ & 30 & 11,4 \\
$5-6$ & 29 & 12,2 \\
7 y más & 140 & 59,3 \\
No dato & 10 & 4,2 \\
TOTAL & 236 & 100,0 \\
\hline
\end{tabular}

En el Cuadro 4 se observa que los grados más avanzados, que se encontraron con la mayor frecuencia, ocurrieron en las pacientes pertenecientes a las décadas de los 31 a los 60 años de edad.

El Cuadro 5 permite apreciar los distintos tratamientos que se emplearon en estas pacientes. En la mayor parte de los casos se empleó el Radium y Rayos X, debido a lo avanzado de las lesiones. En los casos en que se usó uno solo de los procedimientos anteriores, esto se debió a que las pacientes no regresaron a terminar el tratamiento completo.
En el Cuadro 6 se puede ver que no tuvieron ningún seguimiento más de la mitad de las pacientes y que fue menor de un año en otra cuarta parte. Se definió como no seguimiento el hecho de que al revisar la historia de la paciente no se encontrara ninguna nota evolutiva después de

\section{CUADRO 5}

\section{TRATAMIENTO DE 236 PACIENTES CON CARCINOMA DE CUELLO UTERINO.}

Hospital Universitario San Vicente de Paúl 1954-1959 - J. Botero y F. Barros

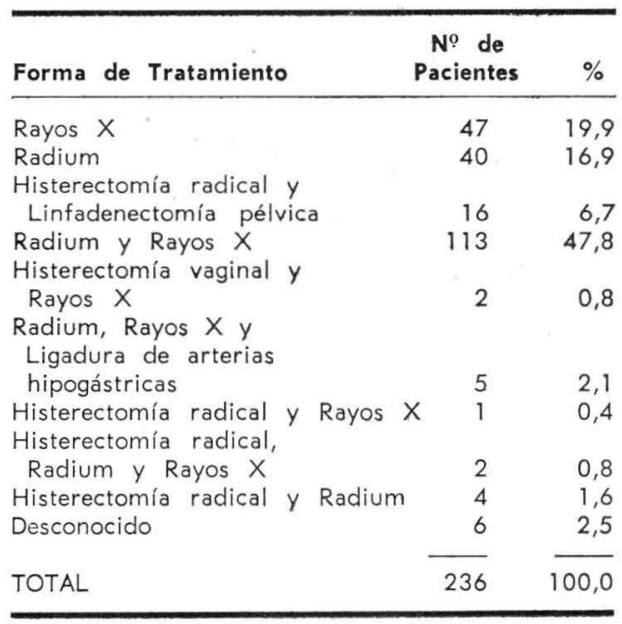

\section{CUADRO 4}

RELACION PORCENTUAL ENTRE LA EDAD Y EL GRADO CLINICO EN 236 PACIENTES CON CARCINOMA DE CUELLO UTERINO.

HOSPITAL UNIVERSITARIO SAN VICENTE DE PAUL. - 1954-1959 - J. Botero y F. Barros

\begin{tabular}{|c|c|c|c|c|c|c|c|c|c|}
\hline $\begin{array}{l}\text { Grados } \\
\text { Grupos }\end{array}$ & $\begin{array}{l}\text { Clínicos } \\
\text { de Edades }\end{array}$ & $\mathrm{N}^{o}$ & Desconocido & 0 & 1 & II & III & IV & $\begin{array}{c}\text { Total } \\
\%\end{array}$ \\
\hline 21 & $-\quad 30$ & 14 & 7,1 & & 7,1 & 35,7 & 42,8 & 7,1 & 100 \\
\hline 31 & -40 & 59 & 10,1 & & 10,1 & 20,3 & 57,6 & 1,6 & 100 \\
\hline 41 & -50 & 81 & 4,9 & & 4,9 & 34,5 & 51,8 & 3,7 & 100 \\
\hline 51 & -60 & 59 & & & 10,1 & 20,3 & 67,7 & 1,6 & 100 \\
\hline $61-$ & -70 & 19 & 5,2 & & 10,5 & 36,8 & 47,3 & & 100 \\
\hline $70 y$ & más & 3 & & & & & 66,6 & 33,3 & 100 \\
\hline \multicolumn{2}{|c|}{ Desconocida } & 1 & & & & & 100,0 & & 100 \\
\hline \multicolumn{2}{|c|}{ TOTALES } & 236 & & & & & & & 100 \\
\hline
\end{tabular}


la admisión de la paciente para recibir el tratamiento que se le había prescrito.

\section{CUADRO 6}

SEGUMIENTO DE LAS PACIENTES CON DIAGNOSTICO DE CANCER DE CUELLO UTERINO.

\begin{tabular}{lrrr}
\multicolumn{4}{c}{ Hospital Universitario San Vicente de Paúl } \\
1954-1959- J. Botero y F. Barros \\
\hline Pacientes seguidas hasta por: & No & $\%$ \\
\hline 1 mes & 127 & 53,8 \\
12 meses ( 1 año) & 59 & 24,4 \\
36 meses ( 3 años) & 18 & 7,6 \\
60 meses (5 años) & 10 & 4,2 \\
84 meses ( 7 años) & 7 & 2,9 \\
108 y más meses & & \\
(9 o más años) & 5 & 1,9 \\
Desconocido & 10 & 4,2 \\
TOTAL & 236 & 100,0 \\
\hline
\end{tabular}

\section{Comentarios}

A pesar de los esfuerzos que se hicieron en este período con el fin de seguir adecuadamente las pacientes tratadas para carcinoma de cuello uterino en sus diferentes estados clínicos, no fue posible hacerlo. Por lo tanto, el dato más importante que se buscaba, cual era el del resultado del tratamiento medido por la supervivencia a los 5 años, no pudo obtenerse. Se considera que el esfuerzo realizado se perdió casi totalmente al no saberse si fueron tratadas en forma adecuada. Los hallazgos mencionados han servido de base para reorganizar la consulta oncológica del servicio de Ginecología, con miras a obtener un funcionamiento más ágil que permita un seguimiento más adecuado de las pacientes. Los detalles de esta nueva organización y los resultados obtenidos en los primeros cinco años de funcionamiento serán evaluados en un próximo futuro. Hay naturalmente factores culturales y económi$\cos$ que influyen en la poca asiduidad con que las pacientes acuden a los controles después del tratamiento, y que no podrán modificarse por lo menos en un futuro próximo.

El resto de la información obtenida permite confirmar que el carcinoma de cuello uterino es una enfermedad primordialmente de grandes multíparas y que las pacientes que asisten a nuestra consulta lo hacen cuando la enfermedad se ha manifestado con síntomas muy evidentes y el grado clínico es por consiguiente muy avanzado.

\section{Resumen}

Se presenta el análisis de algunos datos obtenidos de las historias de 236 pacientes con carcinoma de cuello uterino tratadas en el Hospital Universitario San Vicente de Paúl entre los años de 1954 y 1959. Tal número es una muestra representativa del total de pacientes tratadas en ese período. Solamente el $4,2 \%$ de las pacientes fue seguido por un período hasta de 5 años, lo cual es un número muy escaso para determinar la eficacia de los tratamientos empleados en los diferentes grados clínicos.

\section{Summary}

The histories of 236 patients on whom the diagnosis of carcinoma of the cervix had been made and were treated at the Hospital Universitario San Vicente de Paúl in Medellin, Colombia, between 1954 and 1959 are studied and some data are analyzed. They are a significant sample of the total amount which was treated in that period. The most important information which was obtained, the 5 year follow-up of the patients, is so low, that an appraisal of the eficacy of the treatment in the diferent clinical stages can not be made. 
Vol. $\mathrm{XX}$

$\mathrm{N}^{0} 6$

\section{AGRADECIMIENTOS}

Agradecemos muy sinceramente al Dr. Luis Fernando Duque y al Dr. William Mejía V., ambos de la Escuela de Salud Pública, las orientaciones metodológicas que nos dieron para la elaboración del presente trabajo.

$* * *$

\section{BIBLIOGRAFIA}

1 Ministerio de Salud Pública. Sección de Educación Sanitaria. Boletín del Comité Nacional de Lucha contra el Cáncer. № 14. Página 13. Junio 1965.
2 Departamento Administrativo Nacional de Estadística, D.A.N.E.: Boletín Mensual de Estadística No 215. Febrero 1969.

3 MONTOYA y FLOREZ, J. B.: El cáncer en Antioquia. Revista Clínica. 2: 200 (Jun.), 1917.

4 Departamento Administrativo de Planeación. Dirección Departamental de Estadística. Anuario Estadístico de Antioquia. 197.

5 CORREA, P.: Estudio estadístico del Cáncer en Antioquia. Antioquia Méd. 5: 589, 1955.

6 RESTREPO, J., OCTOA, L. C. y LOPEZ, J. : El problema del cáncer en Medellín. Antioquia Méd. 6: 325, 1956. 